\title{
Survival and growth of 31 Populus clones in South Carolina
}

\author{
David R. Coyle ${ }^{\mathrm{a}, *}$, Mark D. Coleman ${ }^{\mathrm{a}}$, Jaclin A. Durant ${ }^{\mathrm{b}, \mathrm{c}}$, Lee A. Newman ${ }^{\mathrm{b}, \mathrm{d}}$ \\ ${ }^{a}$ USDA Forest Service, Southern Research Station, P.O. Box 700, New Ellenton, SC 29809, USA \\ ${ }^{\mathrm{b}}$ Arnold School of Public Health, Department of Environmental Health Sciences, University of South Carolina, 800 Sumter Street, Columbia, SC 29208, USA \\ ${ }^{\mathrm{c}}$ Department of Entomology, University of California-Riverside, Riverside, CA 92521, USA ${ }^{1}$ \\ ${ }^{\mathrm{d}}$ University of Georgia Savannah River Ecology Laboratory, Drawer E, Aiken, SC 29802, USA
}

Received 21 November 2004; received in revised form 29 July 2005; accepted 8 August 2005

Available online 18 April 2006

\begin{abstract}
Populus species and hybrids have many practical applications, but clonal performance is relatively undocumented in the southeastern United States outside of the Mississippi River alluvial floodplain. In spring 2001, 31 Populus clones were planted on two sites in South Carolina, USA. The sandy, upland site received irrigation and fertilization throughout the growing season, while the bottomland site received granular fertilizer yearly and irrigation in the first two years only. Over three growing seasons, tree survival and growth differed significantly among clones at both sites. Hybrid clones I45/51, Eridano, and NM6 had very high survival at both sites, while pure eastern cottonwood ( $P$. deltoides) clones consistently had the lowest survival. Nearly all mortality occurred during the first year. The $P$. deltoides clone WV416 grew well at both sites, $P$. deltoides clones S13C20 and Kentucky 8 grew well at the bottomland site, and hybrids $184-411$ and 52-225 grew well at the upland site. Based on both survival and growth, clones 311-93, S7C15, 184-411, and WV416 may warrant additional testing in the upper coastal plain region of the southeastern US. Kentucky 8 and S13C20 had excellent growth rates, but initial survival was low. However, this was likely due to planting stock quality. We emphasize this is preliminary information, and that clones should be followed through an entire rotation before large-scale deployment.
\end{abstract}

Published by Elsevier Ltd.

Keywords: Cottonwood; Diameter; Hybrid; Irrigation; Stem volume

\section{Introduction}

Intensively managed forest systems have potential to partially offset the ever-increasing global demand for wood and wood products [1,2]. These forest plantations have tremendous potential for bioenergy production [3], and can also be grown commercially for paper and wood products, furniture, flooring, and shipping material [4]. Fast-growing Populus selections perform extremely well if given proper

\footnotetext{
This research was conducted on official Government time, and therefore the right of the U.S. Government to retain copyright is accepted. The use of trade names in this publication is solely for reader information and does not imply endorsement by the U.S. Department of Agriculture or the University of South Carolina.

*Corresponding author. Current address: Department of Entomology, 345 Russell Laboratories, University of Wisconsin, Madison, WI 53706, USA. Tel.: + 16082624755 ; fax: + 16082623322 .

E-mail address: dcoyle@entomology.wisc.edu (D.R. Coyle).

${ }^{1}$ Current address.
}

silvicultural treatment, including site preparation, weed and pest control, irrigation, and fertilization [5]. Populus also can be used effectively in phytoremediation plantings [6] and for municipal waste treatment [7,8]. Mean annual increment production of seven [9], nine [10], and 25 [11] $\mathrm{Mgha}^{-1} \mathrm{yr}^{-1}$ has been attained in the northeast, northcentral, and northwestern US, respectively. Although numerous trials have taken place in the Lower Mississippi River alluvial floodplain [12-17], limited published information exists regarding Populus performance in other areas of the southeastern US. Yields of nearly $20 \mathrm{Mgha}^{-1} \mathrm{yr}^{-1}$ have been reported in Florida [18], demonstrating the potential of intensively managed Populus in the southeastern US.

Breeding programs develop more genotypes than are selected and propagated for large-scale use [19,20]. From a population of $>10,000$ genotypes, breeders normally attempt to select 5-10 for further testing (R.S. Zalesny, personal communication). Most genotypes are eliminated 
through several screening processes and field trials, which have been done extensively in the northwestern, northcentral, and northeastern US $[9,19,21]$. Populus breeding and selection has also been done to develop clones specifically for the southeastern US [22]. Growth and productivity often garner the majority of attention when screening Populus clones for use in intensively managed systems [1,23-25], but survival is another important selection criterion [26]. Low survival decreases stand yield, and double-planting to mitigate these effects requires extra time and resources. Clones having low survival (as the result of poor rooting) or growth rates are not economically feasible in intensive forestry, and these factors must be considered before Populus genotypes are chosen for largescale deployment in a particular area [19,27]. In addition, variables such as insect and disease resistance and cold tolerance should be considered throughout a full rotation. Small-scale field trials and careful monitoring prior to large-scale deployment are an excellent way to measure these variables [28].

The key to maximizing Populus productivity is to match specific clones to sites on which they perform the best [24]; e.g. clones requiring a long growing season are not suited for use in the northern US, and drought-tolerant clones are best suited for arid regions. Furthermore, Mead [20] contends that proper genotype selection can lead to the greatest gains in intensively managed forest productivity. Our objectives were to identify possible Populus selections suitable for use in this region of the southeastern US. Data from this study will improve our knowledge of several Populus selections' early rotation performance on two different types of sites and soil in South Carolina.

\section{Materials and methods}

\subsection{Study locations}

The experiment was conducted on a bottomland (DArea) and an upland (SRWC) site within the U.S. Department of Energy Savannah River Site, a National Environmental Research Park, located near Aiken, South Carolina, USA $\left(33^{\circ} 23^{\prime} \mathrm{N}, 81^{\circ} 40^{\prime} \mathrm{E}\right)$. The climate is humid continental, with warm, dry summers and mild winters (average temperature: $16.8^{\circ} \mathrm{C}$ ). Study sites were very flat (both surface slopes $<6 \%$ ) with similar soil $\mathrm{pH}(5.1$ at $\mathrm{D}$ Area and 5.5 at SRWC). SRWC was located approximately $15 \mathrm{~km}$ north of D-Area. Annual rainfall at D-Area was 88,103 , and $162 \mathrm{~cm}$ over the 2001-2003 study period, while SRWC rainfall was 66,90 , and $126 \mathrm{~cm}$ during the same time. D-Area soils are classified as Udorthent, with soils of the Blanton (thermic Grossarenic Paleudults), Troup (thermic Grossarenic Paleudults), and Wagram (thermic Arenic Paleudults) series throughout the study area [29]. These soils are composed of a sandy clay loam at the surface, followed by several $\mathrm{cm}$ of sandy material and $10-12 \mathrm{~cm}$ of sandy loam. Previous vegetation was mature loblolly pine (Pinns taeda L.), harvested in fall 2000.
Stumps were removed and slash was cleared after harvesting. The Blanton series of sandy and well-drained soils (to a depth of $120 \mathrm{~cm}$ ) is predominant at SRWC [30]. Previous vegetation was mature $P$. taeda and longleaf pine ( $P$. palustris L.). Specific D-Area and SRWC site characteristics are outlined in WSRC [29] and Coleman et al. [31], respectively.

\subsection{Plant material}

A total of 18 and 31 Populus clones (comprised of both pure species and hybrids) were planted at the D-Area and SRWC study sites, respectively (Table 1). Clones were obtained from commercial nurseries, government research programs, industry programs, and university personnel. Clones chosen represented a range of growing regions and genotypes, with particular emphasis on those reported or expected to do well in the southeastern US. Dormant hardwood cuttings (size range $20-60 \mathrm{~cm}$ in length, $0.9-1.9 \mathrm{~cm}$ diameter) were stored at $3{ }^{\circ} \mathrm{C}$ and soaked in water $48 \mathrm{~h}$ prior to planting to promote optimum rooting [32].

\subsection{Study design and treatments}

The D-Area site was planted on 30 March 2001. Four blocks were scattered throughout the 2 ha study site. Block 1 was approximately $30 \mathrm{~m}$ from block 2 , both of which were approximately $0.5 \mathrm{~km}$ from block 3 . Block 4 was approximately $1 \mathrm{~km}$ from any other block. Eighteen clonal plots were planted in each block: clones S7C15, 52-225, and Eridano were planted adjacent to each other in randomly ordered 36-tree plots ( 6 rows of 6 trees); the other 15 clones were planted in 16-tree plots (4 rows of 4 trees) randomly arranged throughout the remaining block area. Clones were planted at $1.3 \times 1.3 \mathrm{~m}$ spacing. Two border rows (a $P$. deltoides clone of unknown origin) surrounded each block to reduce edge effects [33]. During the 2001 and 2002 growing seasons, two blocks at D-Area received irrigation at a rate of 1.5 and $2.0 \mathrm{~cm} \mathrm{wk}^{-1}$, respectively, while control plots received $0.1 \mathrm{~cm} \mathrm{wk}^{-1}$ irrigation. Trees received only rainwater in 2003. Granular fertilizer $\left(112 \mathrm{~kg} \mathrm{Nha}^{-1}\right)$ plus micronutrients was applied to all blocks each spring.

The SRWC site was planted on 22 April 2001, and contained two blocks, each having 31 clonal plots. Due to material availability, clones 110531, 112127, ST70, ST71, ST109, and ST260 were planted in eight-tree plots ( 2 rows of 4 trees); all others were planted in 16-tree plots. Trees were planted at $2.5 \times 2.5 \mathrm{~m}$ spacing. Two border rows of an unknown $P$. deltoides clone surrounded each block. Trees on the SRWC site were irrigated $3.0 \mathrm{~cm} \mathrm{wk}^{-1}$ and fertilized yearly at a rate of $160 \mathrm{~kg} \mathrm{Nha}^{-1}$ from time of establishment (2001) or 1 April (2002 and 2003) until 30 September.

We applied oxyflourfen (Goal 2XL ${ }^{\circledR}$, Rohm and Haas, Philadelphia, PA) in the spring and glyphosate (Roundup ${ }^{\mathbb{R}}$ 
Table 1

Populus clones evaluated between 2001 and 2003 on two sites in South Carolina

\begin{tabular}{|c|c|c|}
\hline Clone & Parentage & Origin $^{a}$ \\
\hline 110531 & P. deltoides & Bolivar Co., MS \\
\hline $110804^{\mathrm{b}}$ & P. deltoides & Bolivar Co., MS \\
\hline 112127 & P. deltoides & Claiborne Co., MS \\
\hline 112830 & P. deltoides & Wilkinson Co., MS \\
\hline $7300502^{\mathrm{b}}$ & P. deltoides & Pulaski Co., IL \\
\hline $7302801^{\mathrm{b}}$ & P. deltoides & Alexander Co., IL \\
\hline $\mathrm{D} 105^{\mathrm{a}}$ & P. deltoides & Osceola Co., WI \\
\hline Kentucky $8^{\mathrm{b}}$ & P. deltoides & Unknown KY county \\
\hline $\mathrm{S} 13 \mathrm{C} 20^{\mathrm{b}}$ & P. deltoides & Miller Co., AR \\
\hline $\mathrm{S} 7 \mathrm{C} 1^{\mathrm{b}}$ & P. deltoides & Brazos Co., TX \\
\hline $\mathrm{S} 7 \mathrm{C} 15^{\mathrm{b}}$ & P. deltoides & Brazos Co., TX \\
\hline ST $66^{\mathrm{b}}$ & P. deltoides & Issaquena Co., MS \\
\hline ST70 & P. deltoides & Issaquena Co., MS \\
\hline ST71 & P. deltoides & Issaquena Co., MS \\
\hline ST109 & P. deltoides & Bolivar Co., MS \\
\hline ST260 & P. deltoides & Brazos Co., TX \\
\hline ST261 & P. deltoides & Brazos Co., TX \\
\hline ST264 & P. deltoides & Unknown eastern TX county \\
\hline WV $99^{\mathrm{b}}$ & P. deltoides & Issaquena Co., MS \\
\hline WV $316^{\mathrm{b}}$ & P. deltoides & Issaquena Co., MS \\
\hline WV415 & P. deltoides & Washington Co., MS \\
\hline WV $416^{\mathrm{b}}$ & P. deltoides & Washington Co., MS \\
\hline WV $94^{\mathrm{b}}$ & $P$. deltoides $\times P$. deltoides & Issaquena Co., MS $\times$ Issaquena Co., MS \\
\hline Eridano $^{\mathrm{b}}$ & $P$. deltoides $\times P$. maximowiczii & Unknown parent from France $\times$ unknown parent from Japan \\
\hline $\mathrm{NM}^{\mathrm{b}}$ & $P$. nigra $\times P$. maximowiczii & Unknown parent clone origins, bred in Germany \\
\hline $\mathrm{I} 45 / 51^{\mathrm{b}}$ & P. deltoides $\times P$. nigra & Unknown parent clone origins, bred in Italy \\
\hline OP367 & $P$. deltoides $\times P$. nigra & Unknown parent clone origins, bred in Oxford Co., ME \\
\hline $15-29^{\mathrm{b}}$ & $P$. trichocarpa $\times P$. deltoides & Chi 80-1 (Chilliwack, BC, Canada) × STl (Issaquena Co., MS) \\
\hline $184-411$ & P. trichocarpa $\times P$. deltoides & RAN 91-568 (Cowlitz Co., WA) × OK 17-10 (Morton Co., KS) \\
\hline $52-225$ & P. trichocarpa $\times P$. deltoides & GF 93-968 (Snohomish Co., WA) × ILL 101 (Perry Co., IL) \\
\hline $311-93$ & P. trichocarpa $\times P$. nigra & NIS 8-1046 (Pierce Co., WA) $\times$ unknown (Loire Valley, France) \\
\hline
\end{tabular}

${ }^{a}$ "Hybrid origin designation follows the pattern "female parent clone (origin) $\times$ male parent clone (origin)".

${ }^{b}$ Clone was planted at both D-Area and SRWC study sites (denoted "common clones").

PRO, Monsanto Corp., St. Louis, MO) as needed according to label directions for weed control. Granular lime was applied to both sites to raise soil $\mathrm{pH}$ to 6.5 .

\subsection{Growth measurements}

Total tree survival was recorded in fall each year. Basal diameter (recorded at $10 \mathrm{~cm}$ to avoid error associated with stump swell) and height were recorded yearly during dormancy on all trees. Aboveground stem volume (hereafter referred to as "volume") was calculated as diame$\operatorname{ter}^{2} \times$ height. To facilitate tree growth comparisons among years and to use the aforementioned volume calculation, we measured basal diameter, not $\mathrm{DBH}$, because not all trees had reached $1.37 \mathrm{~m}$ at the conclusion of the first growing season.

\subsection{Statistical analyses}

Due to uneven sample sizes $(8,16$, or 36 tree plots) survival was weighted to reflect the number of observations, $n$, that went into the computation of each survival proportion [34] using the equations

$v=p(1-p / n)$

and

$w=1 / \sqrt{(v)}$,

where the weight, $w$, for each proportion, $p$, was the reciprocal of the square root of the variance, $v$.

Survival at the conclusion of the 2001 and 2003 growing seasons was analyzed using a repeated measures ANOVA (Proc Mixed, SAS Inc., Cary, NC). At D-Area, we were most interested in the effects of clone, irrigation, and the clone $\times$ irrigation interaction to determine if clones receiving irrigation experienced greater survival. The year and clone $\times$ year and clone $\times$ irrigation $\times$ year interactions also were examined to determine if clone survival was more influenced by rootability (indicated by the 2001 measurement) or pests and site adaptability (indicated by the 2003 measurement). Since all trees at the SRWC site received the same treatment, we were only interested in differences among clones and years, as well as the clone $\times$ year interaction. Finally, we compared clones at D-Area 
receiving the irrigation treatment with the same clones at SRWC (hereafter referred to as "common clones"; Table 1) to examine the effects of site, clone, year, and the site $\times$ clone and clone $\times$ year interactions on survival.

Basal stem diameter is well correlated with stem volume in several tree species [35] including Populus [36,37]. Since stem volume from 3-yr-old Populus has been shown to be an excellent selection criterion [15] and correlates well with 7-yr-old stem volume [16], we only report stem diameter and volume. Height may [38] or may not [39] be well correlated with Populus productivity, thus lending uncertainty to its use as a selection criterion.

Diameter and volume from 2001 to 2003 were analyzed using an ANOVA with year as the repeated measure (Proc Mixed, SAS Inc., Cary, NC). The effects of clone, irrigation, the clone $\times$ irrigation, clone $\times$ year (to determine if overall clonal growth rankings changed throughout the years), and clone $\times$ irrigation $\times$ year (to determine if either the irrigated or control clones experienced changes in ranking over the years) interactions were examined. Clone and the clone $\times$ year interaction were examined at the SRWC site. Common clones were tested for the effects of site, clone, and site $\times$ clone interactions.

Mean separations were performed using Tukey's $t$-test $(\alpha=0.05)$. Only irrigated WV416 clones survived at DArea; therefore, this clone is only included in the survival and common clone analyses. A single ST70 tree survived at the SRWC site; data for this clone was not included in any growth analysis because the lack of competition most likely led to an increased growth rate in this tree.

Table 2

Mean survival and rankings for all clones combined across irrigation treatments at D-Area after three growing seasons

\begin{tabular}{llc}
\hline Clone & $2003 \pm$ SE & Rank \\
\hline I45/51 & $72 \pm 20$ & 1 \\
Eridano & $59 \pm 14$ & 2 \\
NM6 & $58 \pm 18$ & 3 \\
ST66 & $58 \pm 13$ & 4 \\
15-29 & $57 \pm 10$ & 5 \\
S7C15 & $56 \pm 12$ & 6 \\
WV99 & $50 \pm 7$ & 7 \\
WV94 & $45 \pm 15$ & 8 \\
WV416 & $36 \pm 21$ & 9 \\
7300502 & $36 \pm 9$ & 10 \\
WV316 & $36 \pm 11$ & 11 \\
WV415 & $34 \pm 14$ & 12 \\
S13C20 & $34 \pm 5$ & 13 \\
110804 & $33 \pm 4$ & 14 \\
Kentucky 8 & $31 \pm 11$ & 15 \\
S7C1 & $27 \pm 12$ & 16 \\
7302801 & $25 \pm 8$ & 17 \\
D105 & $25 \pm 7$ & 18 \\
\hline
\end{tabular}

Clonal rankings did not differ between 2001 and 2003. Means sharing a line are not significantly different (Tukey's HSD; $\alpha=0.05$ ).

\section{Results}

\subsection{Survival}

$D$-Area. Survival differed significantly among clones $(P<0.0001$; Table 2$)$, and ranged from $27 \%$ to $96 \%$ and $25 \%$ to $72 \%$ after 2001 and 2003 , respectively. Four of the five clones with the highest survival were hybrids. The clone $\times$ irrigation interaction was significant $(P<0.005)$, as clones S7C1 and WV416 had significantly higher survival when receiving irrigation. No effects on survival were found for irrigation $(P=0.28)$, year $(P=0.92)$, or the clone $\times$ year $(P=0.94)$, and clone $\times$ irrigation $\times$ year interaction $(P=0.98)$.

$S R W C$. Significant survival differences occurred among clones $(P<0.0001)$. With the exception of $\mathrm{S} 7 \mathrm{C} 15$, five hybrids had significantly higher survival than all other clones (Table 3). There was a much wider range of survival (7-99\%) compared to D-Area. There was neither a significant effect of year $(P=1.00)$ nor a clone $\times$ year interaction $(P=1.00)$ as all mortality at SRWC occurred in 2001 as rooting failure.

Table 3

Mean survival and rankings at SRWC after three growing seasons

\begin{tabular}{|c|c|c|c|}
\hline Clone & $\%$ survival $\pm \mathrm{SE}$ & Rank & \\
\hline $\mathrm{I} 45 / 51$ & $99 \pm 0$ & 1 & \\
\hline NM6 & $96 \pm 2$ & 2 & \\
\hline Eridano & $96 \pm 2$ & 2 & \\
\hline $311-93$ & $96 \pm 2$ & 2 & \\
\hline OP367 & $96 \pm 2$ & 2 & \\
\hline $\mathrm{S} 7 \mathrm{C} 15$ & $91 \pm 2$ & 6 & \\
\hline WV416 & $81 \pm 0$ & 7 & \\
\hline ST260 & $75 \pm 7$ & 8 & \\
\hline $184-411$ & $72 \pm 2$ & 9 & \\
\hline $52-225$ & $70 \pm 5$ & 10 & \\
\hline 7300502 & $69 \pm 4$ & 11 & \\
\hline $15-29$ & $64 \pm 3$ & 12 & \\
\hline WV94 & $63 \pm 7$ & 13 & \\
\hline 112127 & $59 \pm 6$ & 14 & 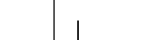 \\
\hline WV99 & $56 \pm 7$ & 15 & \\
\hline Kentucky 8 & $52 \pm 6$ & 16 & 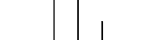 \\
\hline 7302801 & $50 \pm 4$ & 17 & \\
\hline 110531 & $50 \pm 7$ & 17 & \\
\hline ST261 & $50 \pm 3$ & 17 & \\
\hline ST71 & $50 \pm 7$ & 17 & t \\
\hline ST109 & $47 \pm 3$ & 21 & 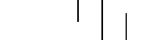 \\
\hline WV415 & $41 \pm 5$ & 22 & \\
\hline ST264 & $41 \pm 3$ & 22 & | \\
\hline $\mathrm{S} 13 \mathrm{C} 20$ & $34 \pm 2$ & 24 & \\
\hline D105 & $33 \pm 10$ & 25 & \\
\hline 110804 & $31 \pm 0$ & 26 & \\
\hline ST66 & $31 \pm 0$ & 26 & \\
\hline $\mathrm{S} 7 \mathrm{C} 1$ & $19 \pm 0$ & 28 & \\
\hline 112830 & $19 \pm 4$ & 28 & \\
\hline WV316 & $16 \pm 5$ & 30 & \\
\hline ST70 & $7 \pm 3$ & 31 & \\
\hline
\end{tabular}

Clonal rankings did not differ between 2001 and 2003. Means sharing a line are not significantly different (Tukey's HSD; $\alpha=0.05$ ). 
Table 4

Mean D-Area volume $\left(\mathrm{dm}^{3}\right)$ accumulation and rankings over three growing seasons

\begin{tabular}{|c|c|c|c|c|c|c|c|c|c|c|c|c|}
\hline \multirow[t]{2}{*}{ Clone } & \multicolumn{6}{|c|}{ Irrigated } & \multicolumn{6}{|c|}{ Non-irrigated } \\
\hline & 2001 & Rank & 2002 & Rank & $2003 \pm \mathrm{SE}$ & Rank & 2001 & Rank & 2002 & Rank & $2003 \pm \mathrm{SE}$ & Rank \\
\hline $\mathrm{S} 13 \mathrm{C} 20$ & 1.34 & 1 & 11.30 & 2 & $20.79 \pm 6.55 \mathrm{a}$ & 1 & 0.42 & 4 & 2.08 & 5 & $3.87 \pm 1.40 \mathrm{abc}$ & 5 \\
\hline WV416 & 1.29 & 2 & 12.62 & 1 & $20.69 \pm 3.83 \mathrm{a}$ & 2 & $\mathrm{na}^{\mathrm{a}}$ & 18 & $\mathrm{na}^{\mathrm{a}}$ & 18 & $\mathrm{na}^{\mathrm{a}}$ & 18 \\
\hline Kentucky 8 & 1.29 & 2 & 10.06 & 3 & $17.54 \pm 4.38 \mathrm{ab}$ & 3 & 0.06 & 13 & 1.23 & 7 & $2.22 \pm 1.21 \mathrm{~cd}$ & 12 \\
\hline 7300502 & 0.61 & 9 & 7.12 & 9 & $15.96 \pm 7.30 \mathrm{ab}$ & 4 & 0.19 & 8 & 0.53 & 14 & $0.91 \pm 0.22 \mathrm{~d}$ & 14 \\
\hline $\mathrm{S} 7 \mathrm{C} 15$ & 0.77 & 6 & 7.34 & 8 & $14.73 \pm 1.97 b$ & 5 & 0.17 & 9 & 1.12 & 11 & $3.28 \pm 0.85 b c$ & 6 \\
\hline $15-29$ & 0.48 & 12 & 7.68 & 6 & $13.66 \pm 1.54 \mathrm{~b}$ & 6 & 0.47 & 3 & 3.55 & 2 & $7.19 \pm 0.87 \mathrm{a}$ & 1 \\
\hline 7302801 & 0.50 & 11 & 7.92 & 5 & $13.20 \pm 4.42 \mathrm{bc}$ & 7 & 0.01 & 17 & 0.66 & 13 & $3.09 \pm 2.18 \mathrm{bcd}$ & 7 \\
\hline Eridano & 0.42 & 14 & 8.72 & 4 & $12.62 \pm 1.85 b c$ & 8 & 0.62 & 1 & 4.00 & 1 & $6.74 \pm 1.00 \mathrm{a}$ & 2 \\
\hline $\mathrm{I} 45 / 51$ & 0.62 & 8 & 7.63 & 7 & $12.46 \pm 1.99 \mathrm{bc}$ & 9 & 0.48 & 2 & 2.66 & 4 & $4.29 \pm 0.92 \mathrm{ab}$ & 4 \\
\hline WV99 & 0.78 & 5 & 6.15 & 11 & $11.03 \pm 2.40 \mathrm{c}$ & 10 & 0.21 & 6 & 1.16 & 10 & $2.57 \pm 1.35 \mathrm{~cd}$ & 9 \\
\hline WV94 & 1.07 & 4 & 6.18 & 10 & $10.32 \pm 1.99 \mathrm{c}$ & 11 & 0.39 & 5 & 1.48 & 6 & $2.99 \pm 1.22 \mathrm{bcd}$ & 8 \\
\hline WV415 & 0.43 & 13 & 4.90 & 13 & $9.55 \pm 2.84 \mathrm{~cd}$ & 12 & 0.09 & 11 & 1.20 & 8 & $2.35 \pm 1.33 \mathrm{~cd}$ & 11 \\
\hline WV316 & 0.75 & 7 & 5.02 & 12 & $9.36 \pm 2.33 \mathrm{~cd}$ & 13 & 0.13 & 10 & 1.18 & 9 & $2.47 \pm 1.04 \mathrm{~cd}$ & 10 \\
\hline ST66 & 0.39 & 15 & 4.30 & 14 & $8.53 \pm 1.53 \mathrm{~cd}$ & 14 & 0.08 & 12 & 0.74 & 12 & $1.60 \pm 0.34 \mathrm{c}$ & 13 \\
\hline NM6 & 0.13 & 17 & 3.82 & 15 & $7.41 \pm 1.36 \mathrm{~cd}$ & 15 & 0.20 & 7 & 2.73 & 3 & $4.52 \pm 0.75 \mathrm{ab}$ & 3 \\
\hline 110804 & 0.51 & 10 & 3.29 & 16 & $5.28 \pm 1.34 \mathrm{~cd}$ & 16 & 0.02 & 15 & 0.32 & 15 & $0.78 \pm 0.18 \mathrm{~d}$ & 15 \\
\hline $\mathrm{S} 7 \mathrm{Cl}$ & 0.14 & 16 & 1.92 & 17 & $3.90 \pm 1.26 \mathrm{~d}$ & 17 & 0.01 & 16 & 0.21 & 17 & $0.53 \pm 0.08 \mathrm{~d}$ & 16 \\
\hline D105 & 0.09 & 18 & 1.24 & 18 & $1.68 \pm 0.34 \mathrm{~d}$ & 18 & 0.05 & 14 & 0.25 & 16 & $0.37 \pm 0.11 \mathrm{~d}$ & 17 \\
\hline
\end{tabular}

Means sharing a letter within a treatment are not significantly different (Tukey's HSD; $\alpha=0.05$ ).

${ }^{\mathrm{a}}$ na $=$ no trees survived.

Common clones. Survival at SRWC was significantly greater than at $\mathrm{D}$-Area $(P<0.001)$. Significant differences also occurred among clones $(P<0.0001)$, as I45/51, NM6, and Eridano survived better across sites. A significant site $\times$ clone interaction $(P<0.0001)$ indicated that three hybrids (I45/51, Eridano, and NM6) had lower survival at D-Area. Neither the effects of year $(P=0.44)$, the clone $\times$ year $(P=0.99)$ nor site $\times$ clone $\times$ year interactions $(P=0.99)$ affected survival.

\subsection{Growth}

$D$-Area. Growth responses varied widely, as diameter (data not shown; $P<0.0001)$ and volume $(P<0.01)$ differed significantly among clones. Diameter showed the greatest range among $P$. deltoides clones, from $2.23 \pm 0.14 \mathrm{~cm}$ in clone $\mathrm{S} 13 \mathrm{C} 20$ to $4.72 \pm 0.46 \mathrm{~cm}$ in clone D105. Both diameter and volume did not significantly increase due to irrigation alone (all $P=0.13$ ), but there was a significant clone $\times$ irrigation $(P<0.034)$ and clone $\times$ irrigation $\times$ year interaction (all $P<0.0001$; Table 4). Overall, volume was $73 \%$ greater in clones receiving irrigation at the end of the third growing season. Five P. deltoides clones (7300502, 110804, S7C1, WV99, and Kentucky 8) each accumulated at least $76 \%$ more stem volume when irrigated (Fig. 1). The clone $\times$ year interaction was significant for diameter and volume (all $P<0.0001$ ), signifying a change in clonal rankings over time (Table 4).

SRWC. Diameter (data not shown) and volume (Table 5) differed significantly among clones at the SRWC site (all $P<0.0001)$. After three growing seasons diameter ranged from $3.07 \pm 2.33 \mathrm{~cm}$ in clone D105 to $7.06 \pm 4.38 \mathrm{~cm}$ in clone 184-411. A significant clone $\times$ year interaction $(P<0.0001)$ indicated changing rankings as clones aged (Table 5). Certain clones, such as 52-225 and ST264, appeared to be slower-growing trees early on, but increased their growth rate as they developed (Table 5). Others, such as Eridano and $\mathrm{S} 7 \mathrm{C} 1$, exhibited the opposite pattern.

Common clones. Neither site (all $P=0.76$ ) nor the site $\times$ clone interaction (all $P=0.28$ ) significantly affected diameter (data not shown) or volume, whereas clonal effects were significant (all $P<0.0001$; Table 6). Pure $P$. deltoides clone WV416 produced over 10 times more stem volume than another $P$. deltoides clone, D105 (Table 6), further highlighting the broad production range exhibited when testing large numbers of clones. Ten of the 12 largest clones were at D-Area receiving irrigation, and clones grown here were $31 \%$ larger than their SRWC counterparts.

\section{Discussion}

\subsection{Survival}

Survival is important for intensively managed Populus production [26]. Clones at our study sites exhibited a wide range of survival (Tables 2 and 3). This is common when evaluating large numbers of clones [10,40-42] due to broad clonal variation in most traits, although some studies have recorded high overall survival rates $[9,43]$. While some studies in the southeastern US have shown $P$. deltoides survival rates $>80 \%[14,16,17,44]$; Stanturf et al., unpublished data], others, including this study, found either a 


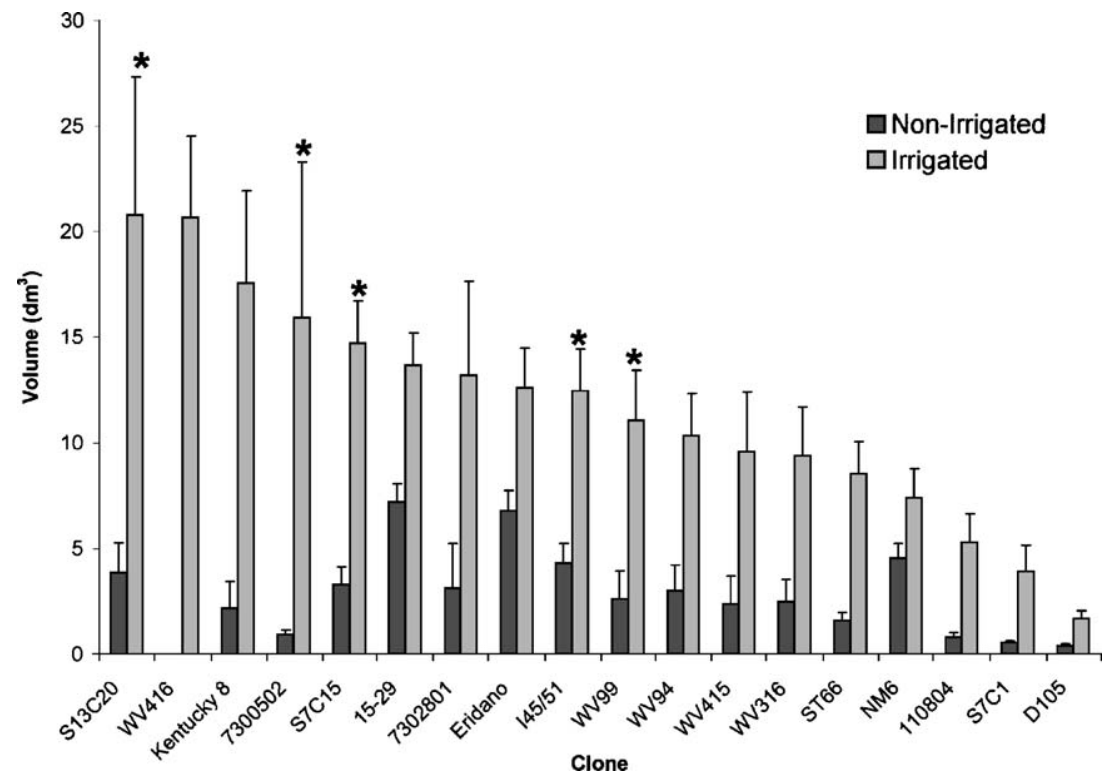

Fig. 1. Three-year-old stem volume (mean \pm SE) of irrigated and non-irrigated Populus clones at D-Area. Within a clone, a "*" indicates a significant difference between non-irrigated and irrigated trees $(\alpha=0.05)$.

Table 5

Mean SRWC volume $\left(\mathrm{dm}^{3}\right)$ accumulation and rankings over three growing season

\begin{tabular}{|c|c|c|c|c|c|c|}
\hline Clone & 2001 & Rank & 2002 & Rank & $2003 \pm S E$ & Rank \\
\hline $184-411$ & 0.93 & 1 & 29.53 & 1 & $31.55 \pm 4.83 \mathrm{a}$ & 1 \\
\hline WV416 & 0.71 & 2 & 14.15 & 2 & $25.59 \pm 3.03 b$ & 2 \\
\hline $52-225$ & 0.37 & 9 & 9.68 & 6 & $17.61 \pm 1.87 \mathrm{c}$ & 3 \\
\hline $\mathrm{S} 13 \mathrm{C} 20$ & 0.38 & 6 & 10.68 & 5 & $15.85 \pm 2.86 \mathrm{~cd}$ & 4 \\
\hline $311-93$ & 0.58 & 4 & 13.61 & 3 & $14.87 \pm 1.51 \mathrm{~cd}$ & 5 \\
\hline Kentucky 8 & 0.34 & 11 & 7.94 & 7 & $14.44 \pm 1.72 \mathrm{~cd}$ & 6 \\
\hline $15-29$ & 0.38 & 7 & 12.32 & 4 & $14.28 \pm 2.02 \mathrm{~cd}$ & 7 \\
\hline ST264 & 0.17 & 21 & 5.80 & 14 & $13.83 \pm 2.76 \mathrm{cde}$ & 8 \\
\hline 112127 & 0.33 & 12 & 5.31 & 16 & $13.57 \pm 3.41 \mathrm{cdef}$ & 9 \\
\hline WV316 & 0.40 & 5 & 6.42 & 12 & $12.87 \pm 3.30 \mathrm{cdefg}$ & 10 \\
\hline $\mathrm{S} 7 \mathrm{C} 15$ & 0.61 & 3 & 6.80 & 11 & $11.75 \pm 1.41 \mathrm{defg}$ & 11 \\
\hline NM6 & 0.21 & 18 & 7.27 & 8 & $10.26 \pm 1.45 \mathrm{efg}$ & 12 \\
\hline ST260 & 0.35 & 10 & 4.87 & 20 & $9.77 \pm 1.66 \mathrm{efgh}$ & 13 \\
\hline WV94 & 0.30 & 13 & 6.14 & 13 & $9.60 \pm 1.35 \mathrm{efgh}$ & 14 \\
\hline Eridano & 0.37 & 8 & 7.24 & 9 & $9.16 \pm 1.00 \mathrm{fgh}$ & 15 \\
\hline 110531 & 0.17 & 20 & 5.16 & 18 & $8.56 \pm 1.83 \mathrm{fghi}$ & 16 \\
\hline ST261 & 0.21 & 17 & 4.73 & 21 & $8.46 \pm 1.43 \mathrm{fghi}$ & 17 \\
\hline $\mathrm{S} 7 \mathrm{C} 1$ & 0.27 & 14 & 5.21 & 17 & $8.30 \pm 2.90$ fghi & 18 \\
\hline WV99 & 0.27 & 15 & 5.07 & 19 & $8.28 \pm 1.11 \mathrm{fghi}$ & 19 \\
\hline 7300502 & 0.13 & 24 & 3.75 & 23 & $7.97 \pm 1.24 \mathrm{ghi}$ & 20 \\
\hline OP367 & 0.24 & 16 & 6.99 & 10 & $7.92 \pm 0.86 \mathrm{ghi}$ & 21 \\
\hline 7302801 & 0.10 & 27 & 2.89 & 25 & $7.45 \pm 1.90 \mathrm{ghi}$ & 22 \\
\hline ST71 & 0.19 & 19 & 3.41 & 24 & $7.01 \pm 1.72 \mathrm{ghi}$ & 23 \\
\hline $\mathrm{I} 45 / 51$ & 0.11 & 25 & 5.53 & 15 & $6.78 \pm 0.71 \mathrm{ghi}$ & 24 \\
\hline 110804 & 0.16 & 22 & 4.11 & 22 & $6.55 \pm 1.03 \mathrm{ghi}$ & 25 \\
\hline ST66 & 0.05 & 29 & 2.40 & 27 & $6.08 \pm 2.17 \mathrm{ghi}$ & 26 \\
\hline WV415 & 0.11 & 26 & 2.69 & 26 & $4.61 \pm 0.66 \mathrm{hi}$ & 27 \\
\hline 112830 & 0.14 & 23 & 1.47 & 29 & $3.10 \pm 0.72 \mathrm{i}$ & 28 \\
\hline ST109 & 0.04 & 30 & 1.23 & 30 & $2.93 \pm 0.66 \mathrm{i}$ & 29 \\
\hline D105 & 0.06 & 28 & 1.75 & 28 & $2.66 \pm 0.60 \mathrm{i}$ & 30 \\
\hline
\end{tabular}

Means sharing a letter are not significantly different (Tukey's HSD; $\alpha=0.05)$.
Table 6

Mean common clone volume $\left(\mathrm{dm}^{3}\right)$ accumulation and rankings over three growing seasons

\begin{tabular}{lcrrrlr}
\hline Clone & 2001 & Rank & 2002 & Rank & $2003 \pm$ SE & Rank \\
\hline WV416 & 0.98 & 1 & 13.43 & 1 & $23.29 \pm 2.41 \mathrm{a}$ & 1 \\
S13C20 & 0.90 & 2 & 11.01 & 2 & $18.52 \pm 3.75 \mathrm{~b}$ & 2 \\
Kentucky 8 & 0.80 & 3 & 8.97 & 4 & $15.94 \pm 2.27 \mathrm{bc}$ & 3 \\
15-29 & 0.45 & 8 & 9.09 & 3 & $13.85 \pm 1.22 \mathrm{c}$ & 4 \\
S7C15 & 0.71 & 4 & 7.14 & 6 & $13.62 \pm 1.35 \mathrm{~cd}$ & 5 \\
Eridano & 0.40 & 9 & 8.15 & 5 & $11.28 \pm 1.21 \mathrm{~d}$ & 6 \\
7300502 & 0.27 & 15 & 4.73 & 13 & $10.29 \pm 2.31 \mathrm{~d}$ & 7 \\
WV316 & 0.67 & 5 & 5.34 & 11 & $10.16 \pm 1.94 \mathrm{~d}$ & 8 \\
WV94 & 0.66 & 6 & 6.16 & 8 & $9.94 \pm 1.16 \mathrm{~d}$ & 9 \\
7302801 & 0.27 & 14 & 5.03 & 12 & $9.79 \pm 2.14 \mathrm{~d}$ & 10 \\
WV99 & 0.52 & 7 & 5.60 & 10 & $9.63 \pm 1.30 \mathrm{~d}$ & 11 \\
I45/51 & 0.35 & 11 & 6.51 & 7 & $9.49 \pm 1.07 \mathrm{~d}$ & 12 \\
NM6 & 0.18 & 16 & 5.88 & 9 & $9.12 \pm 1.03 \mathrm{~d}$ & 13 \\
ST66 & 0.29 & 12 & 3.74 & 15 & $7.81 \pm 1.25 \mathrm{e}$ & 14 \\
WV415 & 0.29 & 13 & 3.94 & 14 & $7.41 \pm 1.67 \mathrm{e}$ & 15 \\
110804 & 0.35 & 10 & 3.66 & 16 & $5.86 \pm 0.86 \mathrm{e}$ & 16 \\
S7C1 & 0.18 & 17 & 2.86 & 17 & $5.00 \pm 1.23 \mathrm{ef}$ & 17 \\
D105 & 0.07 & 18 & 1.47 & 18 & $2.12 \pm 0.34 \mathrm{f}$ & 18 \\
\end{tabular}

Means sharing a letter are not significantly different (Tukey's HSD; $\alpha=0.05)$.

wide range of survival or overall poor survival [13,45]. With the exception of Krinard and Kennedy [14], all of the aforementioned studies having high survival rates initially double- or triple-planted cuttings at each location, then thinned all but the most vigorous tree. Had we employed this method, it is likely that our overall survival would have been higher. 
The lack of a significant year effect indicated that most (D-Area) or all (SRWC) mortality occurred during the 2001 growing season. In general, survival was greater at DArea compared to SRWC. Populus trees are hydrophilic, but standing water may lower growth rates and in some cases result in death. Some flooding that occurred at DArea in 2001 may have decreased survival in some clones. Furthermore, because we obtained our clonal material from a wide variety of sources, it was impossible to standardize cutting size or vigor. Cutting size [32] and location source [46] have been shown to impact tree survival and growth; cuttings of some clones appeared healthier than others in our study (DRC, personal observation). Collecting cutting material after allowing all clones to grow in a common nursery for one year prior to trial initiation would have eliminated this variance. We were unable to statistically evaluate differences among effects of source nursery and cutting size on growth rates and survival in our study.

Hybrids survived much better than pure species in our study. Hybrid clones I45/51, OP367, 311-93, Eridano, and NM6 all had $>95 \%$ survival at SRWC, and high hybrid survival rates are not uncommon in other clone evaluation studies. Survival of six [9] and 54 [40] hybrid Populus clones grown in New York ranged from $89-99 \%$ and $63-100 \%$, respectively, while survival of 11 hybrid Populus clones ranged from 47-96\% in Maryland [47]. NM6 survival was $>94 \%$ in New York [41] and Wisconsin [21], and 87\% in Maryland [47]. Thus, it appears that NM6 survives well in a wide range of geographic regions, an excellent characteristic for selections used in intensively managed forest systems.

Three common clones with lower survival at D-Area were hybrids (Eridano, I45/51, and NM6). Soil quality and composition may have influenced survival, as soils were sandier and more porous at the SRWC site, allowing for better drainage. Conversely, the shallower and finer soil texture at D-Area may have held too much water near the surface, and this, along with early season flooding, may have caused anaerobic stress in some clones. Site variability can exert a large impact on Populus survival and productivity $[10,48]$, and matching clones to sites where they perform best is crucial to maximizing productivity.

\subsection{Growth}

The positive effect of irrigation on Populus growth is well established, as trees in this genus are hydrophilic [45,49,50]. Irrigation positively affected Populus growth at both DArea (Fig. 1) and at the SRWC site [31]. Volume gains up to $1600 \%$ were realized in pure $P$. deltoides clones, while all hybrids gained over $63 \%$ (Fig. 1). The pure P. deltoides clones that showed a significant growth increase with irrigation were S13C20, Kentucky 8, WV99, and S7C15, all of southern US origin. Populus are facultative wetland species, naturally existing on mesic sites in this region, which may indicate why these clones performed so poorly without irrigation.

Soil conditions may have contributed to greater overall growth among irrigated clones at D-Area. Soils at D-Area had higher clay content than those at SRWC and retained water better. Even though the SRWC site received more consistent irrigation, soils here had lower water holding capacity, lower water table, and greater porosity, all characteristics that likely decreased soil water availability. Regardless, certain clones performed well at both sites. When testing large numbers of clones, it often becomes difficult to separate out each clone from another; rather, clones settle out into groups of superior, moderate, and poor performing clones $[9,21,41,51]$. For example, clones S13C20, Kentucky 8, S7C15, and WV416 each grew well on both study sites over the three seasons monitored, indicating better adaptability. In addition, clones such as 184-411 and 52-225 would warrant further testing due to their high growth rates on the SRWC site. The ST70 tree had $72 \%$ more stem volume than the next largest clone in the study (184-411) but this high growth rate observed in the lone survivor was probably due to the lack of competition. Stem volume is positively correlated to available growing space in some tree species [52], but we found no relationship between clone survival rank and volume rank at D-Area $\left(R^{2}=0.003\right)$ or SRWC $\left(R^{2}=0.011\right)$. Clones 112830 and $\mathrm{S} 7 \mathrm{Cl}$, also having extremely low survival, failed to exhibit increased growth rates as did ST70. If growth of ST70 was typical, and initial survival could be improved, then this clone could have potential for use in intensive forest management in the upper coastal plain of the southeastern US. Alternatively, clones D105 (a top performer in the Lake States) [53], 110804, and ST66 (which did not perform well at either site), and clones S7C1, ST109, 112830, and ST71 (which did not perform well at the SRWC site) may not warrant further evaluation on similar study sites.

When evaluating large numbers of selections, it is often useful to assign rankings based on a particular measurement variable, such as survival, growth, or pest susceptibility. Researchers can then further evaluate a predetermined group, such as the top 10 clones. However, due to the vast number of clones that are continually being produced, time is often the limiting factor when evaluating clones. Therefore, short trials-sometimes as short as one growing season - are sometimes used to decide which clones deserve further testing. Unfortunately, clonal rankings often change as stands develop due to variation in disease resistance, response to cold, and rootability [10,27]. Short evaluation trials can falsely indicate clonal performance over a harvest rotation. For instance, clone DN170 initially ranked 11th in growth and disease resistance but ranked third after four additional growing seasons [10]. Clones in our study exhibited similar changes in rank over the first three growing seasons (Tables 5 and 6). In most cases, the clone rankings stayed fairly consistent: the top three clones at each site remain the top three throughout 
the study, with only minimal ranking movement. However, there were instances where clones made large changes upward or downward in the rankings. Between 2001 and 2003, clone volume rankings of 15-29 increased from 10th to 4th at D-Area; rankings for 52-225 exhibited the same upward movement at SRWC over this time period. Other clones exhibited the opposite pattern, such as WV94 at DArea and S7C15 at SRWC.

Populus selections have tremendous productivity potential [24]. Their effectiveness in intensively managed forests and phytoremediation plantings depends on optimizing growth by selecting genotypes that perform well on a particular site. Clones with high initial rankings but whose growth rates decline after a few years may be more suited for use in biomass plantings or coppice rotations [28]. Conversely, clones whose growth rates increase as they age should not be discarded from consideration because their initial growth is slow. Matching a particular clone to a particular growing site takes much data and interpretation; a balance must be struck between survival, growth, and pest susceptibility. While hybrids generally had higher survival, pure $P$. deltoides generally showed greater growth. Based on these data, we have identified several clones that performed well regarding survival and growth. Further testing may be justified on clones 311-93, S7C15, 184-411, and WV416. Also, clones Kentucky 8 and S13C20 may be evaluated further if proper silvicultural techniques were used to ensure initial survival.

\section{Acknowledgments}

We thank the many undergraduate technicians who have assisted with this project: Tucker Slack, Virginia Slater, Luke Williams, LaDawndrea Priest, Rebecca Rose, Cory Hall, and Ginger Jones (USDA Forest Service-Savannah River), and Jessie Brown, Danny Heenan and Meghan Langley (University of Georgia Savannah River Ecology Laboratory and University of South Carolina). This manuscript was greatly improved by the comments of Drs. Ronald Zalesny (USDA Forest Service North Central Research Station, Rhinelander, WI), Richard Hall (Iowa State University, Ames, IA) Randy Rousseau (MeadWestvaco Corp., Wickliffe, KY), John Stanturf (USDA Forest Service Southern Research Station, Athens, GA), and two anonymous reviewers. We thank the various suppliers for providing plant material. Thanks to Dr. John Blake and the members of the USFS-Savannah River Engineering Division for infrastructure work at D-Area. Dr. Stan Zarnoch (USDA Forest Service, Asheville, NC) provided valuable statistical discussions.

\section{References}

[1] Fox TR. Sustained productivity in intensively managed forest plantations. Forest Ecology and Management 2000;138:187-202.
[2] Fenning TM, Gershenzon J. Where will the wood come from? Plantation forests and the role of biotechnology. Trends in Biotechnology 2002;20:291-6.

[3] Parikka M. Global biomass fuel resources. Biomass and Bioenergy 2004:27:613-20.

[4] Stanton B, Eaton J, Johnson J, Rice D, Schuette B, Moser B. Hybrid poplar in the Pacific Northwest. Journal of Forestry 2002;100:28-33.

[5] Stanturf JA, van Oosten C, Netzer DA, Coleman MD, Portwood CJ. Ecology and silviculture of poplar plantations. In: Dickmann DI, Isebrands JG, Eckenwalder JE, Richardson J, editors. Poplar Culture in North America. Ottawa, Canada: National Research Council; 2001. p. 153-206.

[6] Newman LA, Strand SE, Choe N, Duffy J, Ekuan G, Ruszaj M, et al. Uptake and biotransformation of trichloroethylene by hybrid poplars. Environmental Science Technology 1997;31:1062-7.

[7] Abrahamson LP, Robison DJ, Volk TA, White EH, Neuhauser EF, et al. Sustainability and environmental issues associated with willow bioenergy development in New York (USA). Biomass and Bioenergy 1998; 15:17-22.

[8] Isebrands JG, Kamosky DF. Environmental benefits of poplar culture. In: Dickmann DI, Isebrands JG, Eckenwalder JE, Richardson J, editors. Poplar Culture in North America. Ottawa, Canada: National Research Council; 2001. p. 207-18.

[9] Tharakan PJ, Robinson DJ, Abrahamson LP, Nowak CA. Multivariate approach for integrated evaluation of clonal biomass production potential. Biomass and Bioenergy 2001;21:237-47.

[10] Netzer DA, Tolsted DN, Ostry ME, Isebrands JG, Riemenschneider DE, Ward KT. Growth, yield, and disease resistance of 7- to 12-yearold poplar clones in the north central United States. General Technical Report NC-229. St. Paul, MN: U.S. Department of Agriculture, Forest Service, North Central Research Station; 2002. p. 1-31.

[11] Heilman PE, Xie F. Influence of nitrogen on growth and productivity of short-rotation Populus trichocarpa $\times$ Populus deltoides hybrids. Canadian Journal of Forest Research 1993;23:1863-9.

[12] Farmer RE, Wilcox JR. Preliminary testing of eastern cottonwood clones. Theoretical and Applied Genetics 1968;38:197-201.

[13] Randall WK, Krinard RM. First-year growth and survival of long cottonwood cuttings. USDA Forest Service Southern Forest Experiment Station 1977; Research Note SO-222: p. 1-3.

[14] Krinard RM, Kennedy Jr HE. Growth and yields of 5-year-old planted hardwoods on sharkey clay soil. Research Note SO-217. USDA Forest Service Southern Forest Experiment Station 1981; p. 1-3.

[15] Olson JR, Jourdain CY, Rousseau RJ. Selection for cellulose content, specific gravity, and volume in young Populus deltoides clones. Canadian Journal of Forest Research 1985;15:393-6.

[16] Forester GS. Provenance variation of eastern cottonwood in the Lower Mississippi Valley. Silvae Genetica 1986;35:32-8.

[17] Knowe SA, Foster GS, Rousseau RJ, Nance WL. Eastern cottonwood clonal mixing study: predicted diameter equations. Canadian Journal of Forest Research 1994;24:405-14.

[18] Stricker JA, Rockwood DL, Segrest SA, Alker GR, Prine GM, Carter DM. Short rotation woody crops for Florida. In: Proceedings of third biennial conference short rotation woody crops operations working group, 10-13 October 2000, Syracuse, NY. 2001; p. 15-23.

[19] Riemenschneider DE, Stanton BJ, Vallée G, Périnet P. Poplar breeding strategies. In: Dickman DI, Isebrands JG, Eckenwalder JE, Richardson J, editors. Poplar Culture in North America. Ottawa, Canada: National Research Council; 2001. p. 43-76.

[20] Mead DJ. Opportunities for improving plantation productivity. How much? How quickly? How realistic? Biomass and Bioenergy 2005;28:249-66.

[21] Robison DJ, Raffa KF. Productivity, drought tolerance and pest status of hybrid populus: tree improvement and silvicultural implications. Biomass and Bioenergy 1998;14:1-20.

[22] Land Jr SB, Stine M, Ma X, Rockwood DL, Warwell MV, Alken GR. 2001. A tree improvement program for eastern cottonwood in 
the southeastern United States. In: Dean JFD, editor. Proceedings, 26th Biennial Southern Forest Tree Improvement Conference, June 26-29, 2001. Athens, GA. University of Georgia, Georgia Center for Continuing Education. Sponsored Publication no, 48. Southern Forest Tree Improvement Committee; p. 84-93.

[23] Tuskan GA, Rensema TR. Clonal differences in biomass characteristics, coppice ability, and biomass prediction equations among four Populus clones grown in eastern North Dakota. Canadian Journal of Forest Research 1992;22:348-54.

[24] Ceulemans R, Deraedt W. Production physiology and growth potential of poplars under short-rotation forestry culture. Forest Ecology and Management 1999;121:9-23.

[25] Hibbs D, Withrow-Robinson B, Brown D, Fletcher R. Hybrid poplar in the Willamette valley. Western Journal of Applied Forestry 2003; 18:281-5.

[26] Chambers PGS, Borralho NMG. Importance of survival in shortrotation tree breeding programs. Canadian Journal of Forest Research 1997;27:911-7.

[27] Ares A. Changes through time in traits of poplar clones in selection trials. New Forests 2002;23:105-19.

[28] Zsuffa L, Sennerby-Forse L, Weisgerber H, Hall RB. Strategies for clonal Forestry with poplars, aspens, and willows. In: Ahuja MR, Libby WJ, editors. Clonal Forestry. II. Conservation and Application. Berlin: Springer; 1993. p. 91-119.

[29] WSRC. Treatability study work plan for phytoremediation of the DArea TCE-contaminated groundwater plume (U). WSRC-RP-20004015, Revision 1 Redline. Westinghouse Savannah River Company, Savannah River Site, Aiken, South Carolina. 2000; p. 1-37.

[30] Rogers VA. Soil survey of the Savannah River Plant area, parts of Aiken, Barnwell, and Allendale counties, South Carolina. In: USDA Soil Conservation Service, Washington, DC. 1990.

[31] Coleman MD, Coyle DR, Blake J, Britton K, Buford M, Campbell B, et al. Production of short rotation woody crops grown with a range of nutrient and water availability: establishment report and first-year responses. SRS-GTR-072: USDA-Forest Service, Southern Research Station, Asheville, NC. 2004; p. 1-21.

[32] Desroachers A, Thomas BR. A comparison of pre-planting treatments on hardwood cuttings of four hybrid poplar clones. New Forests 2003;26:17-32.

[33] Zavitkovski J. Small plots with unplanted plot border can distort data in biomass production studies. Canadian Journal of Forest Research 1981;11:9-12.

[34] Zar JH. Biostatistical Analysis, 4th ed. Upper Saddle River, NJ: Prentice-Hall; 1999.

[35] Martin JG, Kloeppel BD, Schaefer TL, Kimbler DL, McNulty SG. Aboveground biomass and nitrogen allocation of ten deciduous southern Appalachian tree species. Canadian Journal of Forest Research 1998;28:1648-59.

[36] Pontailler JY, Ceulemans R, Guittet J, Mau F. Linear and non-linear functions of volume index to estimate woody biomass in high density young poplar stands. Annals of Forest Science 1997;54:335-45.

[37] Calfapietra C, Gielen B, Sabatti M, De Angelis P, ScarasciaMugnozza G, Ceulemans R. Growth performance of Populus exposed to "Free Air Carbon dioxide Enrichment" during the first growing season in the POPFACE experiment. Annals of Forest Science 2001;58:819-28.
[38] Knowe SA, Foster GS, Rousseau RJ, Nance WL. Height-age and height-diameter relationships for monocultures and mixtures of eastern cottonwood clones. Forest Ecology and Management 1998;106:115-23.

[39] Walsh TA, Burk TE, Isebrands JG. Development and evaluation of quantitative functions for early selection of Populus clones. Biomass and Bioenergy 1996;11:151-9.

[40] Abrahamson LP, White EH, Nowak CA, Biggs RD, Robison DJ. Evaluating hybrid poplar clonal growth potential in a three-year-old genetic selection field trial. Biomass 1990;21:101-14.

[41] Lo MH, Abrahamson LP. Principal component analysis to evaluate the relative performance of nine year old hybrid poplar clones. Biomass and Bioenergy 1996;10:1-6.

[42] Kiernan BD, Volk TA, Tharakan PJ, Nowak CA, Phillipon SP et al. Clone-site testing and selections for scale-up plantings. Biomass Power for Rural Development Final Report. 2003; p. 1-73.

[43] Kopp RF, White EH, Abrahamson LP, Nowak CA, Zsuffa L, Burns KF. Willow biomass trials in central New York state. Biomass and Bioenergy 1993;5:179-87.

[44] Foster GS, Rousseau RJ, Nance WL. Eastern cottonwood clonal mixing study: intergenotypic competition effects. Forest Ecology and Management 1998;112:9-22.

[45] Lockaby BG, Clawson RG, Baker T. Response of three hardwood species to irrigation and fertilization on an upland site. Southern Journal of Applied Forestry 1997;21:123-9.

[46] Zalesny RS, Hall RB, Bauer EO, Riemenschneider DE. Shoot position affects root initiation and growth of dormant unrooted cuttings of Populus. Silvae Genetica 2003;52:273-9.

[47] Kays JS. Results of hybrid poplar clone trial after two years at the ERCO Tree Farm in Prince George's county, Maryland. University of Maryland Extension Research Note-RNS3. 2002; p. $1-6$.

[48] Hansen EA, Ostry ME, Johnson WD, Tolsted DN, Netzer DA, Berguson WE et al. Field performance of Populus in short-rotation intensive culture plantations in the north-central U.S. Research Paper NC-320. St. Paul, MN: U.S. Department of Agriculture, Forest Service, North Central Forest Experiment Station. 1994; p. 1-13.

[49] Souch CA, Williams S. Growth, productivity and water use in three hybrid poplar clones. Tree Physiology 1998;18:829-35.

[50] Shock CC, Feibert EBG, Seddigh M, Saunders LD. Water requirements and growth of irrigated hybrid poplar in a semi-arid environment in eastern Oregon. Western Journal of Applied Forestry 2002;17:46-53.

[51] Pellis A, Laureysens I, Ceulemans R. Growth and production of a short rotation coppice culture of poplar. I. Clonal differences in leaf characteristics in relation to biomass production. Biomass and Bioenergy 2004;27:9-19.

[52] Pinkard EA, Neilsen WA. Crown and stand characteristics of Eucalyptus nitens in response to initial spacing: implications for thinning. Forest Ecology and Management 2003;172:215-27.

[53] Isebrands JG, Riemenschneider DE. Evaluation of genetic selection criteria for Populus clones: 1995 annual progress report. In: North Central Forest Experiment Station, USDA Forest Service, Rhinelander, WI. U.S. Department of Energy Bioenergy Feedstock Development Program IA \#DE-A105-940R22197; 1996:1-51. 